\title{
Evaluation of pulmonary endothelial function in Fontan patients
}

\author{
Heiner Latus, MD, ${ }^{\mathrm{a}, \mathrm{b}}$ Andrea Lederle, MD, ${ }^{\mathrm{a}}$ Markus Khalil, MD, ${ }^{\mathrm{a}}$ Gunter Kerst, MD, ${ }^{\mathrm{a}, \mathrm{c}}$ \\ Dietmar Schranz, MD, ${ }^{\mathrm{a}}$ and Christian Apitz, $\mathrm{MD}^{\mathrm{a}, \mathrm{d}}$
}

\section{ABSTRACT}

Objectives: Pulmonary endothelial dysfunction due to a loss of pulsatile pulmonary blood flow is thought to be a major contributor in the development of increased pulmonary vascular resistance (PVR) in patients with a Fontan circulation. We sought to evaluate pulmonary endothelial dysfunction by vasodilator response to acetylcholine in children and adolescents with Fontan hemodynamics.

Methods: Twenty-one Fontan patients with a median age of 10.7 years (range, 3.4-30.0 years) underwent invasive cardiac catheterization, including intraarterial Doppler and pressure measurements in a segmental pulmonary artery. Pulmonary flow reserve (PFR) was quantified as the change of average peak velocity in response to acetylcholine infusion. Local PVR was assessed by the ratio of local pressure and blood flow velocity.

Results: Average peak velocity significantly increased from $16.4 \pm 6.6 \mathrm{~cm} / \mathrm{s}$ to $20.1 \pm 6.4 \mathrm{~cm} / \mathrm{s}(P=.002)$ resulting in a mean PFR of $1.28 \pm 0.37$. Local PVR dropped from $0.72 \pm 0.32 \mathrm{~mm} \mathrm{Hg} / \mathrm{cm} / \mathrm{s}$ to $0.57 \pm 0.20 \mathrm{~mm} \mathrm{Hg} / \mathrm{cm} / \mathrm{s}$ $(\mathrm{n}=19 ; P=.01)$. A significant relationship was found between baseline local PVR and PFR $(r=0.73 ; P=.0006)$ as well as between PFR and the percent decrease in local PVR $(r=0.85 ; P<.0001)$. In addition, percentage decrease in local PVR correlated with New York Heart Association functional class, whereas neither PFR nor local PVR correlated with global PVR.

Conclusions: The assessment of PFR and local PVR may help to evaluate adverse pulmonary vascular remodeling in Fontan patients as an adjunct to the usual catheterization protocol, particularly given the difficulties in assessing global PVR in these patients. Future research is required to study whether impaired local pulmonary endothelial function may be associated with Fontan failure. (J Thorac Cardiovasc Surg 2019;158:523-31)

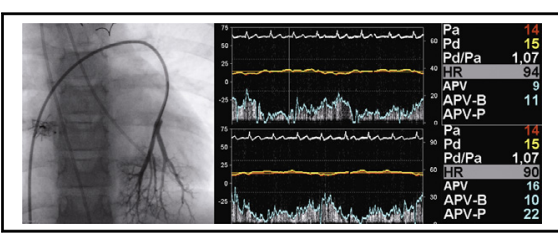

Doppler wire in the lower lobe pulmonary artery for assessment of local vasorelaxation.

\section{Central Message}

Local pulmonary vasodilator response to acetylcholine may help to assess adverse pulmonary vascular remodeling in patients with a Fontan circulation as an adjunct to the usual catheterization protocol

\section{Perspective}

Considering the difficulties in the quantification of global pulmonary vascular resistance in single-ventricle circulation, the assessment of pulmonary flow reserve and local vascular resistance might add information for guiding specific PAH-targeted drug therapy and may carry prognostic information regarding the development of Fontan failure.

See Commentary on page 532.
In the Fontan circulation, blood flow into the lungs occurs in a passive way and depends on low pulmonary vascular resistance (PVR) allowing adequate filling of the single ventricle. ${ }^{1}$ Consequently, increases in PVR can reduce

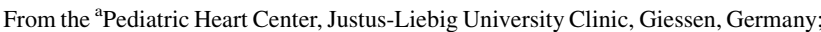
${ }^{\mathrm{b}}$ Department of Pediatric Cardiology and Congenital Heart Disease, German Heart Centre, Munich, Germany; ${ }^{c}$ Department of Pediatric Cardiology, University Children's Hospital, Aachen, Germany; and ${ }^{\mathrm{d}}$ Department of Pediatric Cardiology, University Children's Hospital, Ulm, Germany.

Supported by the German Society of Pediatric Cardiology (DGPK)

Received for publication June 10, 2018; revisions received Nov 8, 2018; accepted for publication Nov 22, 2018; available ahead of print April 4, 2019

Address for reprints: Heiner Latus, MD, Department of Pediatric Cardiology and Congenital Heart Disease, German Heart Centre Munich, Technical University

Munich, Lazarettstr 36, 80636 Munich, Germany (E-mail: heiner.latus@ googlemail.com)

$0022-5223 / \$ 36.00$

Copyright (c) 2019 by The American Association for Thoracic Surgery

https://doi.org/10.1016/j.jtcvs.2018.11.144
}

cardiac output and may ultimately result in a so-called failing Fontan circulation. ${ }^{2}$ Chronic nonpulsatile flow in the pulmonary vascular bed has been suggested to play a major role in the development of adverse pulmonary vascular remodeling in patients with a single ventricle, causing deterioration in the patient's clinical condition. ${ }^{3,4}$

Although pulmonary vasoreactivity testing is a fundamental part in the diagnostic workup of patients with pulmonary hypertension, ${ }^{5-7}$ no systematic protocols are currently available for assessing the response of pulmonary

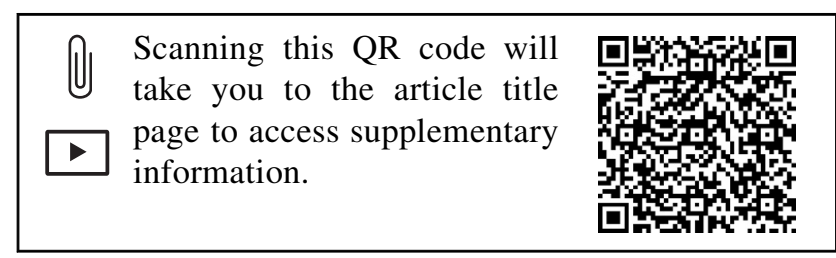




$$
\begin{aligned}
& \text { Abbreviations and Acronyms } \\
& \text { Ach = acetylcholine } \\
& \text { CMR }=\text { cardiovascular magnetic resonance } \\
& \text { mPAP = mean pulmonary artery pressures } \\
& \text { NYHA }=\text { New York Heart Association } \\
& \text { PAH }=\text { pulmonary arterial hypertension } \\
& \text { PCWP }=\text { pulmonary capillary wedge pressure } \\
& \text { PFR }=\text { pulmonary flow reserve } \\
& \text { PVD }=\text { pulmonary vascular disease } \\
& \text { PVR }=\text { pulmonary vascular resistance }
\end{aligned}
$$

vasodilators in single-ventricle patients. ${ }^{2,3,8}$ This is further aggravated by the current lack of a clear definition of pulmonary hypertension and a reliable assessment of PVR in Fontan patients. ${ }^{9}$ Because conventional parameters of global hemodynamics such as cardiac output and PVR are influenced by differences in right-to-left lung perfusion and variable amounts of collateral flow, ${ }^{10}$ a diagnostic modality that allows assessment of pulmonary vascular changes on a local level would be helpful in the evaluation of pulmonary vascular disease (PVD) in these patients.

Vasodilator response to acetylcholine (Ach) has been shown to provide further information regarding the etiology and stage of PVD and has recently been demonstrated to have prognostic relevance in children with idiopathic pulmonary arterial hypertension (PAH). ${ }^{11-16}$ Accordingly, we thought to quantify pulmonary endothelial function by acute vasodilator testing to Ach in Fontan patients undergoing invasive cardiac catheterization to evaluate the role of pulmonary endothelial dysfunction in global Fontan hemodynamic parameters.

\section{MATERIALS AND METHODS \\ Study Population}

Between October 2014 and February 2016, 21 individuals with Fontan circulation ( 8 of whom were female [38\%]) underwent cardiac catheterization as part of their routine clinical assessment at our institution. Indications for catheterization were closure of patent tunnel fenestrations (5 patients), pulmonary arterial interventions (balloon angioplasty in 1 patient and stent implantation in 8 patients), aortic arch interventions ( 2 patients), and diagnostic evaluation in 4 patients. Embolization of aortopulmonary or venovenous collaterals were performed in 7 patients during the procedure. Invasive hemodynamic evaluation also included local pulmonary vasoreactivity testing according to a standardized clinical study protocol established at our institution. Clinical data were retrospectively obtained from hospital medical records, including date of birth, gender, and anatomic diagnoses, as well as age at and type of each surgical procedure. The clinical study protocol was approved by the institutional ethical review board.

\section{Cardiovascular Magnetic Resonance Imaging}

All cardiovascular magnetic resonance imaging (CMR) studies were performed on a 3-Tesla system (Verio; Siemens, Erlangen, Germany). Images were acquired in supine position with two 16-elements phased array coils. Sedation using a combination of propofol and midazolam was applied in patients younger than age 8 years. The CMR protocol included a stack of short-axis slices from the base of the heart to the apex using cine steady-state free precession with breath-hold or gradient echo sequences in free-breathing technique when patients were sedated. Data acquired during breath-hold were assessed with the following sequence parameters: repetition time, $48 \mathrm{~ms}$; echo time, $1.5 \mathrm{~ms}$; flip angle, $60^{\circ}$; slice thickness, $6 \mathrm{~mm}$; in-plane image resolution, $1.3 \mathrm{~mm} \times 1.3 \mathrm{~mm} \times 6.0 \mathrm{~mm}$; and temporal resolution, 25 to 40 phases. In measurements during free-breathing, the sequence parameters were as follows: repetition time, $56 \mathrm{~ms}$; echo time, $2.5 \mathrm{~ms}$; flip angle, $12^{\circ}$; slice thickness, $5 \mathrm{~mm}$; and in-plane image resolution, $1.4 \mathrm{~mm} \times 1.4 \mathrm{~mm} \times 5.0 \mathrm{~mm}$. End-diastolic and end-systolic volume, stroke volume and ejection fractions for the single ventricle were calculated by dedicated software (Argus; Siemens, Erlangen, Germany) after the endocardial borders were traced manually at end-systole and enddiastole. Cardiac output was calculated by multiplying heart rate by stroke volume. All parameters were adjusted to body surface area.

\section{Invasive Hemodynamic Assessment}

Diagnostic cardiac catheterization was performed under local anesthesia through a percutaneous femoral approach using low dosages of midazolam, ketamine, or propofol sedation. Measurements of baseline hemodynamic parameters included venous and arterial saturations, blood gases, mean pulmonary artery pressures (mPAP) and pulmonary capillary wedge pressure (PCWP) in the standard manner with fluid-filled catheters. In patients without a CMR study, blood flow was estimated from the Fick equation and global PVR was calculated from standard equations (mPAP-PCWP/flow). Blood flow and vascular resistances were indexed to body surface area.

\section{Vasodilator Response to Acetylcholine}

Local pulmonary endothelial function was assessed by vasodilator response to Ach before any contrast agent was applied. Dependent on the patient size, a $4 \mathrm{~F}$ or $5 \mathrm{~F}$ multipurpose catheter was inserted into the left lower lobe pulmonary artery (Figure 1). A 0.014-in pulsed ComboWire 9500 (Volcano Corporation, Rancho Cordova, Calif) was positioned through the multipurpose catheter into a straight segment of the medial or posterior branch of the lower lobe pulmonary artery with a diameter of 3 to $5 \mathrm{~mm}$. Position of the ComboWire in the center of this vessel was confirmed by a hand-injection of contrast through a Thouy-Borst adapter (ie, sighting angiography), and a stable flow-velocity signal with minimal noise. Then, serial infusions were made via the multipurpose catheter into the segmental pulmonary artery using an infusion pump with Ach-concentrations of $10^{-6}$ and $10^{-5} \mathrm{M}$, respectively. Simultaneous pressure measurements in the pulmonary artery were performed after calibration of the ComboWire system with routinely used fluid-filled catheters. Changes in pulmonary blood flow in response to Ach were assessed using intravascular Doppler blood flow velocity measurements. Pulmonary flow reserve (PFR) was calculated as the ratio of pulmonary blood flow velocity in response to Ach relative to baseline values. Local PVR was assessed by the ratio of PAP to blood flow velocity (Video 1).

\section{Statistical Analysis}

All continuous variables were tested for normality using the D'Agostino-Pearson omnibus test and are presented as mean \pm standard deviation or median and range, as appropriate. Comparisons of the baseline and Ach data were performed using the $t$ test for paired data or the Wilcoxon matched-pairs signed rank test. Pearson correlation coefficient was used to analyze simple linear relationships between different variables. Analysis was performed using GraphPad statistical software (GraphPad Software Inc, San Diego, Calif).

\section{RESULTS}

\section{Patient Characteristics}

Median age at cardiac catheterization was 10.7 years (range, 3.4-30.0 years) and mean weight was 


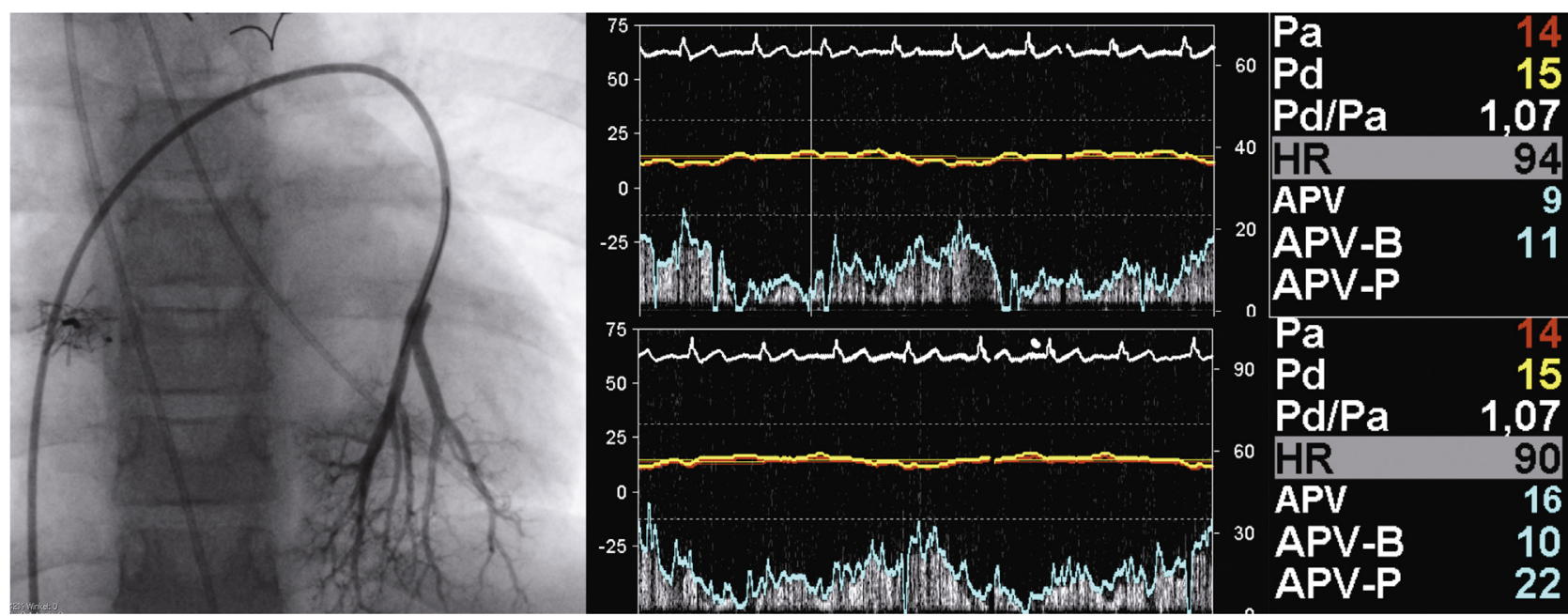

FIGURE 1. Example of local vasoreactivity testing in a Fontan patient. Left, Sighting angiography by hand injection of contrast through a Thouy-Borst adapter to confirm position of the combined Doppler and pressure wire (ComboWire; Volcano Corporation, Rancho Cordova, Calif) in the center of the vessel in anteroposterior projection. The wire was inserted via a multipurpose catheter into the left lower lobe pulmonary artery to achieve a stable flow-velocity signal with minimal noise. Right, Doppler flow signal at baseline (upper panel) and in response to local application of acetylcholine (lower panel). Note the increase in average peak velocity $(A P V)$ from baseline $9 \mathrm{~cm} / \mathrm{s}$ to $16 \mathrm{~cm} / \mathrm{s}$ under acetylcholine infusion. Distal pulmonary artery pressure $(P d)$ (yellow line) was measured simultaneously after calibration of the pressure signal with routinely used fluid-filled catheters $(P a)(r e d ~ l i n e)$. HR, Heart rate.

$35.4 \pm 17.5 \mathrm{~kg}$. Fourteen patients $(67 \%)$ had a morphologic right systemic ventricle and 7 patients $(23 \%)$ had a morphologic left systemic ventricle. Nine patients underwent initial surgical palliation by an aortopulmonary-shunt procedure, whereas in 11 patients a central ( 3 patients) or bilateral (8 patients) pulmonary artery band was inserted to reduce pulmonary blood flow. Median age at total cavopulmonary connection was 3.3 years (range, 2.2-10.0 years) (extracardiac conduit in 18 patients, lateral tunnel in 2 patients, and atriopulmonary connection in 1 patient). Eighteen of the 21 patients $(86 \%)$ had undergone a previous bidirectional cavopulmonary connection. Eight patients were in New York

\section{Local vasodilator response to acetylcholine (Ach)}

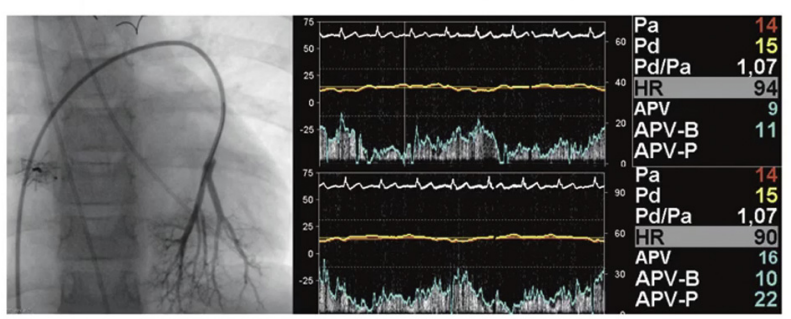

$$
\text { PFR }=A P V_{\text {Ach }} / A P V_{\text {baseline }}
$$

\section{Local PVR = local pressure Pd / APV}

VIDEO 1. Video explaining and discussing the main findings of our study. Video available at: https://www.jtcvs.org/article/S0022-5223(18)33434-2/ fulltext.
Heart Association (NYHA) functional class I, 9 patients in NYHA functional class II, and 4 patients in NYHA functional class III. At the time of catheterization, 2 patients received targeted vasodilator drug therapy with sildenafil and bosentan, respectively, which was suspended 8 hours before catheterization according to our study protocol.

\section{CMR Data}

Of 21 patients, $15(71 \%)$ had a complete CMR study within a time interval of 2.0 months (range, 0-9 months) to cardiac catheterization. Mean indexed end diastolic volume was $78 \pm 24 \mathrm{~mL} / \mathrm{m}^{2}$ and mean indexed end systolic volume was $36 \pm 19 \mathrm{~mL} / \mathrm{m}^{2}$, resulting in an ejection fraction of $57 \% \pm 9 \%$ (Table 1). Mean cardiac index was $3.5 \pm 1.7 \mathrm{~L} / \mathrm{min} / \mathrm{m}^{2}$.

\section{Hemodynamic Parameters Data}

Invasive hemodynamic assessment of the study population showed mPAP $10.6 \pm 2.6 \mathrm{~mm} \mathrm{Hg}$ and PCWP $6.1 \pm 2.5 \mathrm{~mm} \mathrm{Hg}$ resulting in a transpulmonary gradient $1.8 \pm 1.2 \mathrm{~mm} \mathrm{Hg}$. Mean cardiac index assessed by the Fick method in 5 patients was $3.0 \pm 0.8 \mathrm{~L} / \mathrm{min} / \mathrm{m}^{2}$. The resulting global indexed PVR was $1.8 \pm 1.2 \mathrm{WU} \times \mathrm{m}^{2}$ (Table 2).

\section{Local Pulmonary Vasoreactivity Testing}

Average peak velocity significantly increased from $16.4 \pm 6.6 \mathrm{~cm} / \mathrm{s}$ to $20.1 \pm 6.4 \mathrm{~cm} / \mathrm{s}(P=.002)$ resulting in a mean PFR of $1.28 \pm 0.37$ (Figure 2). Distal PAP 
TABLE 1. Demographic, clinical, and cardiovascular magnetic resonance (CMR) data of the study population

\begin{tabular}{|c|c|}
\hline Variable & Result \\
\hline \multicolumn{2}{|l|}{ Clinical data } \\
\hline Male & 13 \\
\hline Female & 8 \\
\hline Weight (kg) & $35.4 \pm 17.5(31$ [13-67]) \\
\hline Body surface area $\left(\mathrm{m}^{2}\right)$ & $1.15 \pm 0.39(1.08[0.58-1.76])$ \\
\hline \multicolumn{2}{|l|}{ Diagnosis } \\
\hline Hypoplastic left heart syndrome & $10(48 \%)$ \\
\hline Tricuspid atresia & $3(14 \%)$ \\
\hline $\begin{array}{l}\text { Atrioventricular septal defect, } \\
\text { unbalanced }\end{array}$ & $3(14 \%)$ \\
\hline Double outlet right ventricle & $3(14 \%)$ \\
\hline $\begin{array}{l}\text { Pulmonary atresia-intact } \\
\text { ventricular septum }\end{array}$ & $1(5 \%)$ \\
\hline $\begin{array}{l}\text { Pulmonary atresia-ventricular } \\
\text { septal defect }\end{array}$ & $1(5 \%)$ \\
\hline \multicolumn{2}{|l|}{ Single ventricle type } \\
\hline Right & $14(67 \%)$ \\
\hline Left & $7(33 \%)$ \\
\hline Age at study (y) & $11.6 \pm 6.1(10.7[3.4-30.0])$ \\
\hline $\begin{array}{l}\text { Age at total cavopulmonary } \\
\text { connection }(\mathrm{y})\end{array}$ & $3.8 \pm 2.0(3.3[2.2-10.0])$ \\
\hline \multicolumn{2}{|l|}{ Initial palliation } \\
\hline Pulmonary artery banding & $11(52 \%)$ \\
\hline Aortopulmonary shunt & $8(38 \%)$ \\
\hline None & $2(10 \%)$ \\
\hline $\begin{array}{l}\text { Previous bidirectional } \\
\text { cavopulmonary connection }\end{array}$ & $18(86 \%)$ \\
\hline \multicolumn{2}{|l|}{$\begin{array}{l}\text { New York Heart Association } \\
\text { functional class }\end{array}$} \\
\hline I & $8(38 \%)$ \\
\hline II & $9(43 \%)$ \\
\hline III & $4(19 \%)$ \\
\hline IV & $0(0 \%)$ \\
\hline $\begin{array}{l}\text { Transcutaneous oxygen } \\
\text { saturation }(\%)\end{array}$ & $93 \pm 3$ (93 [85-99]) \\
\hline Patent fenestration & $5(23 \%)$ \\
\hline \multicolumn{2}{|l|}{ CMR data $(\mathrm{n}=15)$} \\
\hline End diastolic volume* $\left(\mathrm{mL} / \mathrm{m}^{2}\right)$ & $78 \pm 27(76[49-150])$ \\
\hline End systolic volume* $\left(\mathrm{mL} / \mathrm{m}^{2}\right)$ & $35 \pm 21(31[18-105])$ \\
\hline Stroke volume* $\left(\mathrm{mL} / \mathrm{m}^{2}\right)$ & $43 \pm 9(45[28-63])$ \\
\hline Ejection fraction $(\%)$ & $57 \pm 9(58[30-66])$ \\
\hline Cardiac index $\left(\mathrm{L} / \mathrm{min} / \mathrm{m}^{2}\right)$ & $3.5 \pm 1.7(3.0[1.6-8.8])$ \\
\hline
\end{tabular}

Values are presented as $\mathrm{n}$, mean \pm standard deviation, and median (range). $C M R$, Cardiovascular magnetic resonance. *Indexed to body surface area.

remained unchanged (baseline, $10.2 \pm 3.7 \mathrm{~mm} \mathrm{Hg}$ and Ach response, $10.3 \pm 3.6 \mathrm{~mm} \mathrm{Hg} ; P=.87)$. Pressure data were not available in 2 patients due to incorrect pressure calibration. Local PVR dropped from $0.72 \pm 0.32 \mathrm{~mm} \mathrm{Hg} / \mathrm{cm} / \mathrm{s}$ to $0.57 \pm 0.20 \mathrm{~mm} \mathrm{Hg} / \mathrm{cm} / \mathrm{s}(\mathrm{n}=19 ; P=.01)$ (Figure 2). A paradoxical response to Ach with an increase in local PVR was observed in 3 patients (Table E1).
TABLE 2. Data on global hemodynamic parameters and vasodilator response to acetylcholine, invasively assessed by cardiac catheterization

\begin{tabular}{|c|c|}
\hline Variable & Result \\
\hline \multicolumn{2}{|l|}{ Hemodynamic data } \\
\hline $\begin{array}{l}\text { Mean pulmonary artery pressure } \\
(\mathrm{mm} \mathrm{Hg})\end{array}$ & $10.7 \pm 2.7(11[6-16])$ \\
\hline $\begin{array}{l}\text { Pulmonary capillary wedge } \\
\text { pressure }(\mathrm{mm} \mathrm{Hg})\end{array}$ & $6.3 \pm 2.5(5[3-12])$ \\
\hline Transpulmonary gradient $(\mathrm{mm} \mathrm{Hg})$ & $4.4 \pm 1.4(4[2-7])$ \\
\hline $\begin{array}{l}\text { Global indexed pulmonary } \\
\text { vascular resistance }\left(\mathrm{WU} \times \mathrm{m}^{2}\right)\end{array}$ & $1.6 \pm 1.0(1.3[0.5-4.4])$ \\
\hline \multicolumn{2}{|l|}{ Local vasoreactivity testing } \\
\hline $\begin{array}{l}\text { Average peak velocity baseline } \\
(\mathrm{cm} / \mathrm{s})\end{array}$ & $16.6 \pm 6.6(14.1[9.0-33.6])$ \\
\hline $\begin{array}{l}\text { Average peak velocity } \\
\text { acetylcholine }(\mathrm{cm} / \mathrm{s})\end{array}$ & $20.1 \pm 6.4(18.7[11.4-30.8])$ \\
\hline Pulmonary flow reserve & $1.28 \pm 0.37(1.2[0.8-2.3])$ \\
\hline $\begin{array}{l}\text { Distal pulmonary artery pressure } \\
\text { baseline }(\mathrm{mm} \mathrm{Hg})\end{array}$ & $10.2 \pm 3.7(10.0 ; 3.7-20.0)$ \\
\hline $\begin{array}{l}\text { Distal pulmonary artery pressure } \\
\text { acetylcholine }(\mathrm{mm} \mathrm{Hg})\end{array}$ & $10.3 \pm 3.6(10.5 ; 3.9-19.0)$ \\
\hline $\begin{array}{l}\text { Local pulmonary vascular } \\
\text { resistance baseline } \\
(\mathrm{mm} \mathrm{Hg} / \mathrm{cm} / \mathrm{s})\end{array}$ & $0.72 \pm 0.32(0.66[0.25-1.56])$ \\
\hline $\begin{array}{l}\text { Local pulmonary vascular } \\
\text { resistance acetylcholine } \\
(\mathrm{mm} \mathrm{Hg} / \mathrm{cm} / \mathrm{s})\end{array}$ & $0.57 \pm 0.20(0.61[0.19-0.85])$ \\
\hline Heart rate baseline (bpm) & $86 \pm 15(87[61-113])$ \\
\hline Heart rate acetylcholine (bpm) & $87 \pm 16(84[63-116])$ \\
\hline
\end{tabular}

\section{Correlations}

A significant relationship was found between baseline local PVR and PFR $(r=0.73 ; P=.0006)$, local PVR and the percent decrease in local PVR $(r=0.52 ; P=.02)$ (Figure 3) as well as PFR and the percent decrease in local PVR $(r=0.85 ; P<.0001)$. No relationship was found between PFR as well as local PVR with global hemodynamic parameters such as global PVR and cardiac index. No associations were found between PFR, baseline local PVR, and percentage decrease of local PVR with age at study as well as follow-up time after total cavopulmonary connection operation. Transcutaneous oxygen saturations showed a significant inverse relationship with local PVR $(r=-0.60$; $P=.006)$ but were not associated with PFR $(r=-0.37$; $P=.09)$, percent decrease in local PVR $(r=-0.31$; $P=.21)$, or global PVR $(r=0.21 ; P=.38)$.

\section{Influence of NYHA Functional Class}

Patients in NYHA functional class III showed a blunted response to Ach with a median PFR of 1.05 and a paradox median increase in local PVR of $12 \%$ significantly $(P=.03)$ differing from NYHA functional class II patients with a median decrease in local PVR of $22 \%$ (Figure 4 ). No 

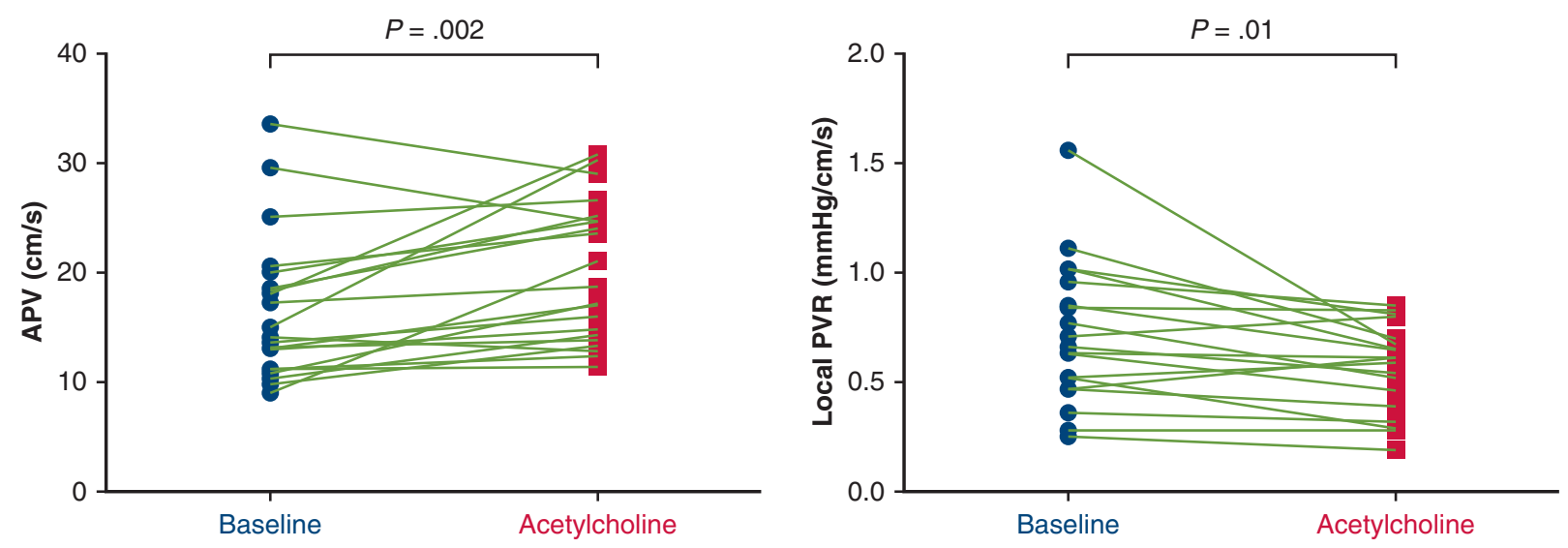

FIGURE 2. Changes in average peak velocity $(A P V)$ and local pulmonary vascular resistance (local $P V R)$ in each individual patient under local pulmonary vasoreactivity testing with acetylcholine. APV increased significantly from $16.4 \pm 6.6 \mathrm{~cm} / \mathrm{s}$ to $20.1 \pm 6.4 \mathrm{~cm} / \mathrm{s}(P=.002)$ and local PVR dropped significantly from $0.72 \pm 0.32 \mathrm{~mm} \mathrm{Hg} / \mathrm{cm} / \mathrm{s}$ to $0.57 \pm 0.20(\mathrm{n}=19)(P=.01)$.

further differences in cardiac index and both global and local PVR were observed between the groups.

\section{Influence of Initial Palliative Procedure}

There was no difference in PFR, local PVR, and decrease in local PVR between patients with initial palliation by an aortopulmonary shunt procedure $(n=9)$ and patients who underwent initial pulmonary artery banding (Figure 5).

\section{DISCUSSION}

To the best of our knowledge, this is the first study assessing PFR as marker of pulmonary endothelial function in Fontan patients. Our results may contribute to the understanding of the development of PVD associated with chronic nonpulsatile pulmonary blood flow as present in the Fontan circulation.

The concept of assessing endothelium-dependent vasorelaxation using Ach has been applied in various experimental and clinical studies investigating endothelial function in the systemic and pulmonary arterial circulation. ${ }^{11,12,17,18}$ Pathologic changes in coronary artery diameter and Doppler wire velocity with Ach injection are suggestive of abnormal epicardial and microvascular coronary vasomotion and may help in the diagnostic workup of patients with ischemic heart disease who present with chest pain but unobstructed arteries. ${ }^{19,20}$ In pulmonary circulation, an intact pulmonary endothelium regulates basal pulmonary-vascular tone and was found to be impaired in patients with various types of PVD. ${ }^{12,14}$ Accordingly, vasodilator response to Ach as an optional measurement adds additional information regarding the etiology and stage of PVD and may help to guide treatment strategies with targeted pulmonary vasodilator drugs. ${ }^{13-15}$

The measured PFR of $128 \%$ of baseline in our patient cohort must be considered abnormal in view of published data in normal controls by other groups using a comparable testing protocol such as that proposed by Celermajer and colleagues ${ }^{12}$ and Kurotobi and colleagues ${ }^{4}$ reporting mean PFRs of $193 \%$ and of $155 \%$, respectively. This finding supports the data of an experimental study by Henaine and colleagues ${ }^{21}$ showing that nonpulsatile pulmonary flow-induced endothelial dysfunction and apoptosis of vascular smooth muscle cells that may result in impaired pulmonary vasodilation, adverse pulmonary vascular remodeling, and elevation of PVR. ${ }^{21}$ In patients with a bidirectional cavopulmonary connection and no supplemental flow through patent pulmonary ventricular outflow, Kurotobi and colleagues ${ }^{4}$ were able to show that impairment in Ach-mediated pulmonary endothelial response was related to the loss of pulmonary artery pulsatility.

In a recent analysis of lung tissue specimens from Fontan patients who died, Ridderbos and colleagues ${ }^{22}$ demonstrated distinct histopathologic features of the intra-acinar pulmonary vessels, including increased intimal fibrosis, disrupted endothelial integrity and loss of vascular smooth muscle cells. All of these findings support the concept of reduced strain and wall shear stress associated with adverse vessel remodeling in the nonpulsatile Fontan circulation. Although no direct relationship was found between transcutaneous oxygen saturation and endothelial function, lower saturations were associated with higher PVR. Chronic hypoxemia may therefore constitute another factor promoting unfavorable vascular remodeling, whereas it remains unknown whether this is a primary effect of hypoxemia or a secondary phenomenon mediated by the presence of aortopulmonary collaterals potentially creating high-flow areas with increased resistance. ${ }^{23}$

Because no associations were found between flow reserve and age and follow-up since Fontan-completion in our population, no time-dependent effects seem to be 

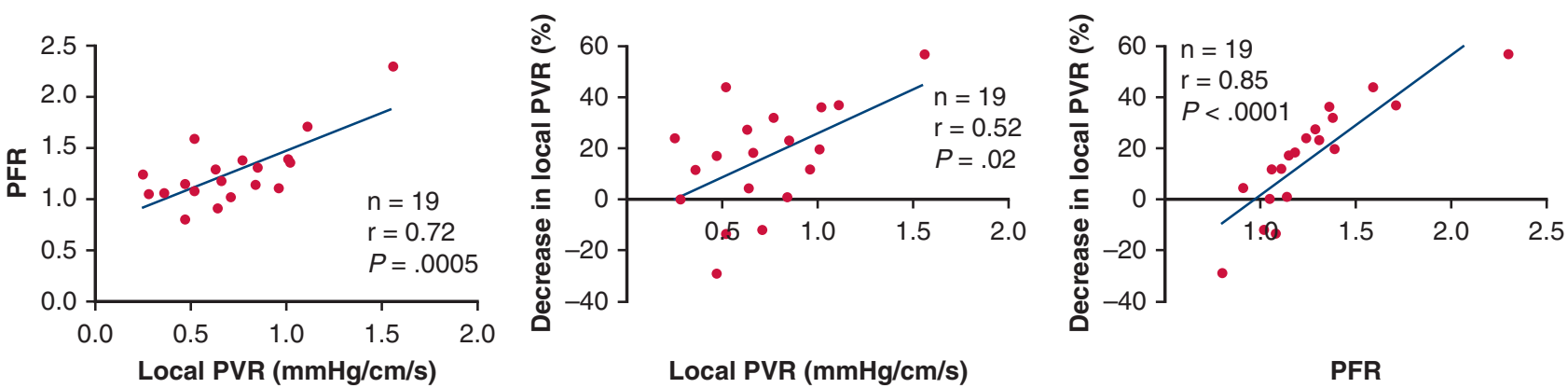

Local PVR $(\mathrm{mmHg} / \mathrm{cm} / \mathrm{s})$

PFR
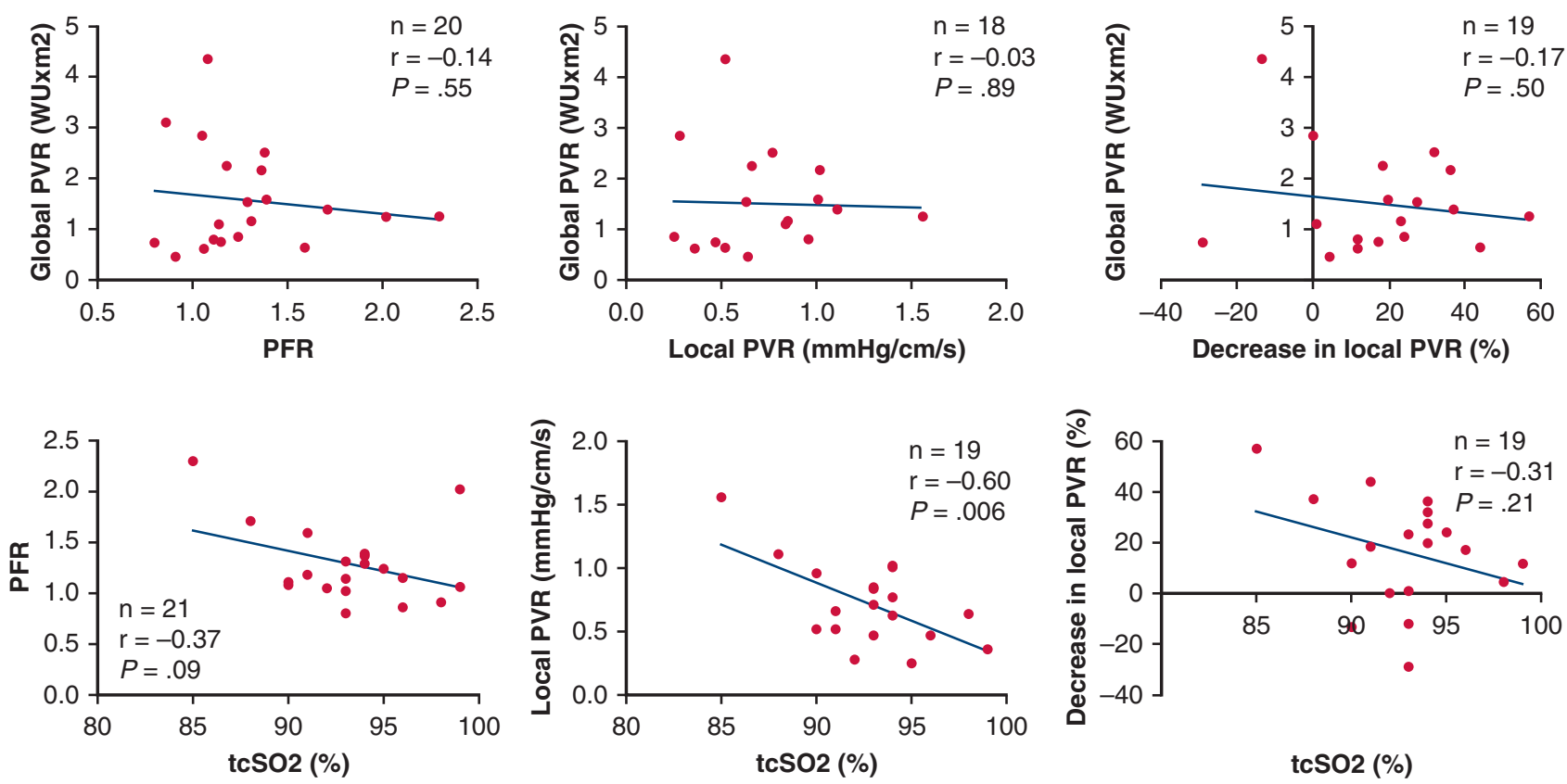

FIGURE 3. Upper panel, Local pulmonary vascular resistance $(P V R)$ was significantly associated with pulmonary flow reserve $(P F R)$ and percentage decrease in local PVR. PFR and percentage decrease in local PVR also showed a significant and strong relationship. Center panel, PFR, local PVR, and percentage decrease in local PVR showed no significant relationship with global pulmonary vascular resistance assessed from routine cardiac catheterization. Lower panel, Transcutaneous oxygen saturations $\left(t c \mathrm{SO}_{2}\right)$ were inversely related to local PVR but not with PFR or percentage decrease in local PVR.

present that accelerate or aggravate the development of pulmonary endothelial dysfunction. Furthermore, initial palliation strategy did not affect pulmonary endothelial function suggesting no relevant long-term influence on pulmonary vascular changes. However, increased pulmonary blood flow through an aortopulmonary shunt may be
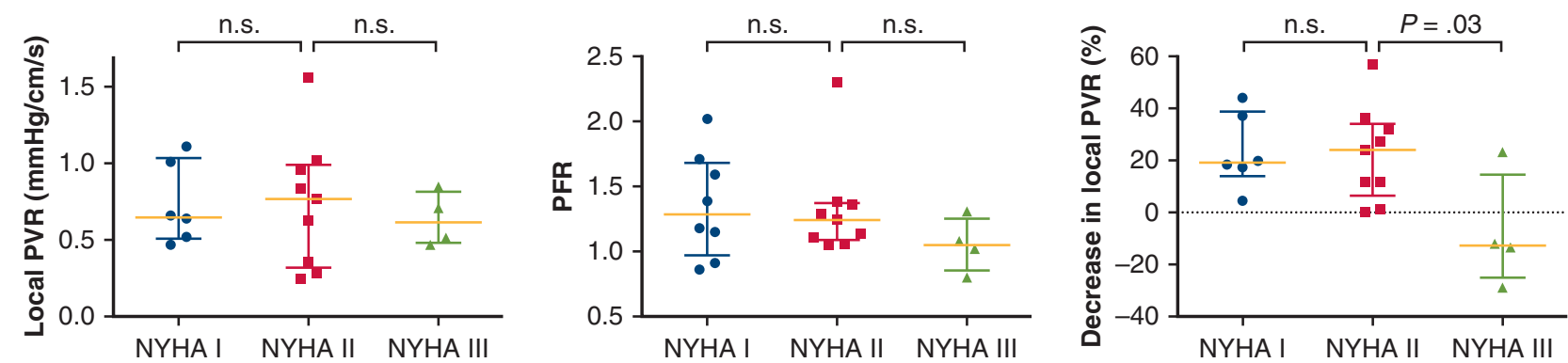

FIGURE 4. Measures of local pulmonary vascular resistance (local PVR), pulmonary flow reserve $(P F R$ ) and decrease in local PVR according to New York Heart Association (NYHA) functional class. Note the paradox response to acetylcholine with an increase in local PVR in 3 of the 4 NYHA functional class III patients. Values are displayed as median (orange line) with interquartile range. n.s., Not significant. 

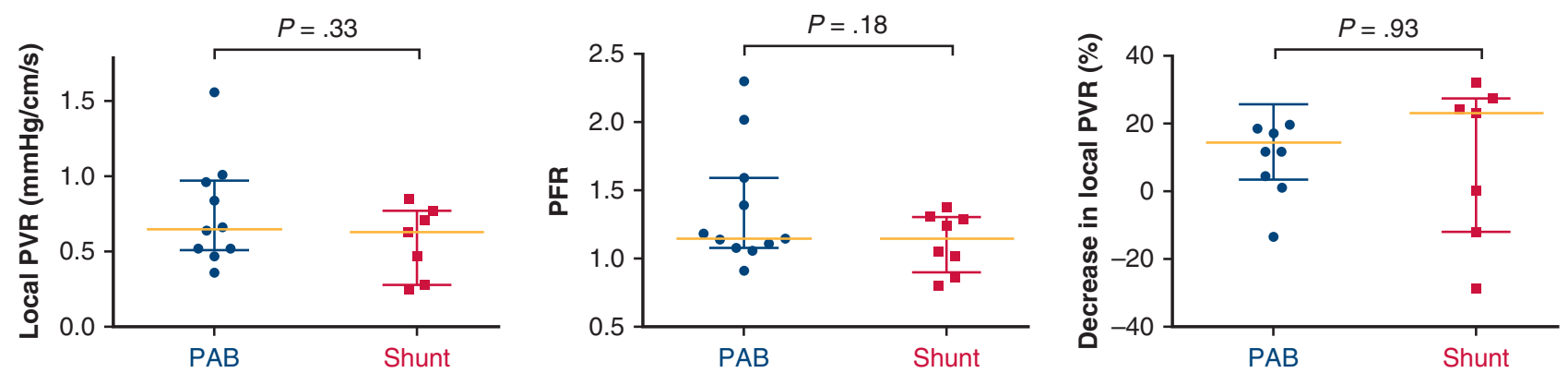

FIGURE 5. No significant differences in local pulmonary vascular resistance (local PVR), pulmonary flow reserve $(P F R)$ and decrease in local PVR were observed between 2 subgroups of patients who underwent initial pulmonary artery banding $(P A B)($ blue $)(\mathrm{n}=11)$ and patients with initial palliation by an aortopulmonary shunt procedure (Shunt) $(r e d)(n=8)$. Values are displayed as median (orange line) with interquartile range.

associated with impaired hemodynamic status directly after Fontan surgery because increased wall thickness of intraacinar vessels has been described in patients who died during or early after the operation. ${ }^{22}$

Compared with patients in favorable functional status, NYHA functional class III patients showed an unexpected increase in local PVR during Ach testing. This finding might be explained by a paradox response of the pulmonary endothelium to Ach, which is an endothelium-dependent vasodilator but can also act as a vasoconstrictor through direct stimulation of muscarinic receptors on vascular smooth muscle cells. ${ }^{24,25}$ Indeed, in 3 of 4 patients in NYHA functional class III, a paradox increase in PAP was observed that resulted in a rise in local PVR. Whether this mechanism is responsible for impairment in exercise capacity and a gradual decline in functional status in Fontan patients remains speculative (methods and results summarized in Figure 6).
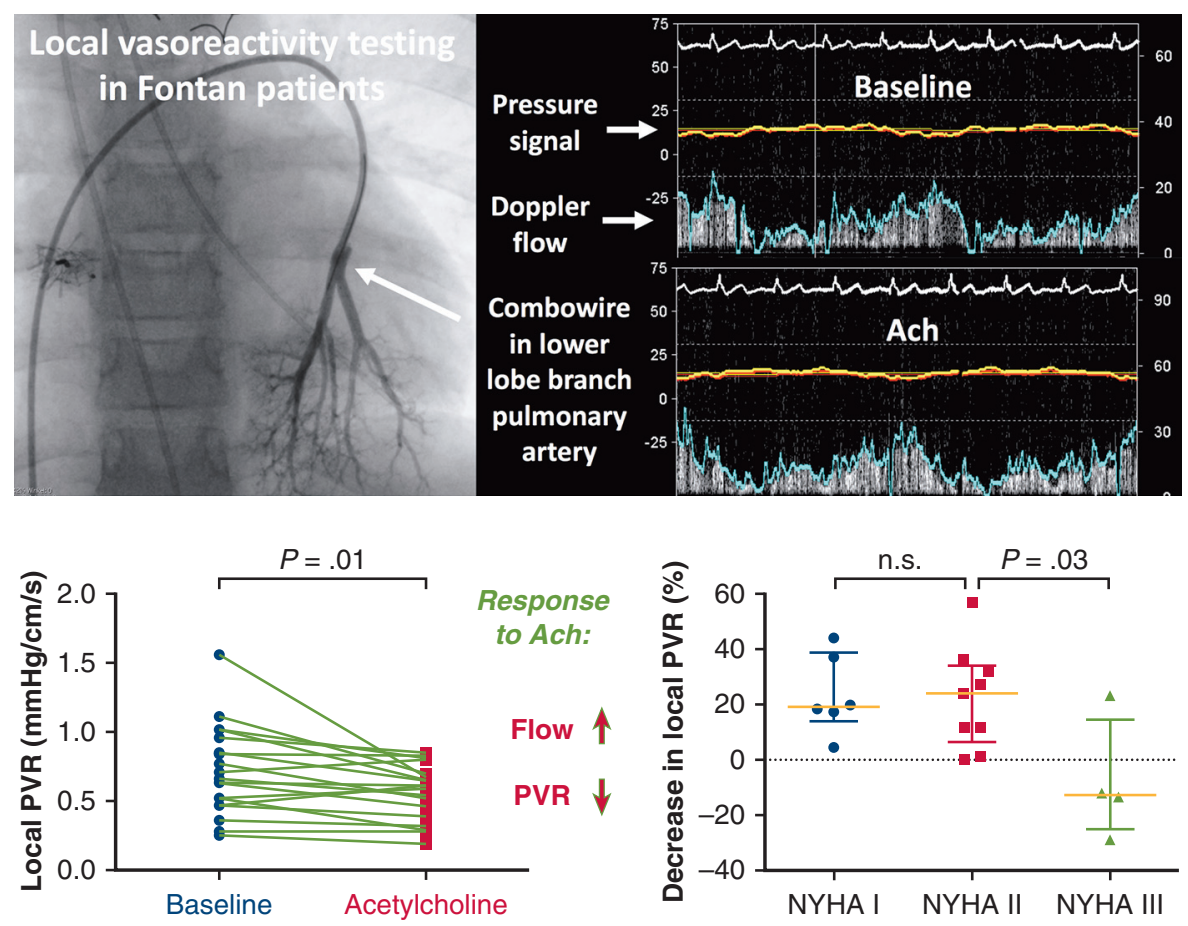

FIGURE 6. The principle and main results of local vasoreactivity testing in Fontan patients. A combined Doppler and pressure wire (ComboWire; Volcano Corporation, Rancho Cordova, Calif) was forwarded into a lower lobe pulmonary artery to assess both local blood flow velocity and pulmonary artery pressure under baseline conditions and after infusion of acetylcholine (Ach). A significant increase in blood flow velocity as a measure of local pulmonary endothelial function (ie, pulmonary flow reserve $[\mathrm{PFR}]$ ) was observed while local pulmonary vascular resistance (PVR) (ie, the ratio of pulmonary artery pressure and blood flow velocity) decreased significantly. Patients in New York Heart Association (NYHA) functional class III showed a pathologic PVR response to Ach with a paradox increase in local PVR in 3 of 4 patients. 
Our findings also demonstrate the limited value of routinely assessed global hemodynamic parameters such as PAP, PVR, and transpulmonary gradient to reflect this adverse remodeling process because these measures showed no relationship with local pulmonary endothelial function and local PVR. The vasodilator response to Ach and the assessment of local PVR might therefore add more reliable information on PVD in Fontan patients because the assessment of global PVR in these patients is prone to error. ${ }^{9,23}$ The reason for this finding might be explained by the fact that endothelial function and local PVR were only measured in 1 single area in the lower pulmonary lobe and therefore might not be representative for the function of the entire pulmonary vascular bed. Additionally, the observed impairment in pulmonary endothelial function might not yet cause a clinically relevant rise in global PVR because the majority of patients showed no relevant rises in mPAP and global $\mathrm{PVR}<3 \mathrm{WU} \times \mathrm{m}^{2}$.

The observed relationship between local PVR and PFR highlights that patients with a higher baseline local PVR had more preserved pulmonary endothelial function. This apparently paradoxical finding illustrates the unique pathophysiology of Fontan circulation and is in accordance with a previous study that found a more profound drop in PVR after inhalation of nitric oxide in Fontan patients influenced by a higher PVR at baseline conditions. ${ }^{3}$ The significant decrease in local PVR in response to Ach is primarily caused by a rise in blood flow velocity, whereas PAP remained unchanged in the majority of patients, which suggests that in the presence of a high baseline flow velocity the response of the endothelium is depressed and is not associated with a further increase in blood flow velocity. This might be due to higher wall shear stress and strain at the pulmonary endothelial cells caused by increased blood flow velocity that not responding to Ach even in the presence of an intact endothelial structure. ${ }^{26,27}$ Accordingly, our findings suggest that the basal pulmonary-vascular tone in Fontan patients with normal PAP and only marginal pulsatility seems primarily regulated by local blood flow velocity.

Our results may also have potential clinical implications: because vasodilator response to Ach is mediated by endothelial nitric oxide, patients with a sustained PFR but elevated local PVR might be good candidates for treatment with PAH-specific medications that are based on modulation of the nitric oxide-cyclic guanosine monophosphate pathway; that is, sildenafil, tadalafil, or riociguat.

\section{Study Limitations}

The number of patients included was small, which limits statistical power and further subgroup analysis. Accordingly, the observed relationships between variables should be primarily considered descriptive and significant correlations do not necessarily imply causality.

Given that no healthy control group with normal pulmonary vascular dynamics was investigated and reliable reference values are not available for patients with singleventricle circulation, further conclusions regarding the degree of attenuation of pulmonary endothelial function are limited. However, in our previous study using an identical vasoreactivity testing protocol a mean PFR of 1.58 (range, 0.5-3.5) was found in children and adolescents with idiopathic PAH. ${ }^{14}$ In this population a PFR $<1.4$ was highly predictive for cardiovascular events. The fact that 16 of 21 Fontan patients had a PFR below that cut-off value supports the hypothesis of abnormal endothelial function in our cohort.

Included patients were referred to our hospital for catheterization due to different indications, which results in a selection bias. However, most of them showed favorable measures of global hemodynamics and were in a stable clinical condition without signs of failing Fontan circulation.

Not all patients tested had a complete CMR study before cardiac catheterization and subsequently calculation of cardiac output and PVR differed methodically in some patients. Furthermore, differences in the used sedation techniques during CMR and invasive assessment may also influence hemodynamic parameter measurements. Hyperemic agents that are typically used to assess microcirculatory resistance in coronary artery circulation such as adenosine were not assessed. ${ }^{28}$ Global vasoreactivity to agents that mediate pulmonary vasodilation by smooth muscle cell relaxation (not endothelium dependent) such as nitroprusside, nitric oxide, or sildenafil was not tested.

\section{CONCLUSIONS}

The assessment of PFR may help to evaluate adverse pulmonary vascular remodeling in Fontan patients as an adjunct to the usual catheterization protocol, particularly given the difficulties in the assessment of global PVR in these patients. Future research is required to study whether impaired local pulmonary endothelial function may be predictive of Fontan failure.

\section{Conflict of Interest Statement}

Authors have nothing to disclose with regard to commercial support.

\section{References}

1. Gewillig M, Brown SC, Eyskens B, Heying R, Ganame J, Budts W, et al. The Fontan circulation: who controls cardiac output? Interact Cardiovasc Thorac Surg. 2010;10:428-33.

2. Van De Bruaene A, La Gerche A, Claessen G, De Meester P, Devroe S, Gillijns H, et al. Sildenafil improves exercise hemodynamics in Fontan patients. Circ Cardiovasc Imaging. 2014;7:265-73.

3. Khambadkone S, Li J, de Leval MR, Cullen S, Deanfield JE, Redington AN. Basal pulmonary vascular resistance and nitric oxide responsiveness late after Fontan-type operation. Circulation. 2003;107:3204-8. 
4. Kurotobi S, Sano T, Kogaki S, Matsushita A, Miwatani T, Takeuchi M, et al. Bidirectional cavopulmonary shunt with right ventricular outflow patency: the impact of pulsatility on pulmonary endothelial function. J Thorac Cardiovasc Surg. 2001;121:1161-8.

5. Hansmann G, Apitz C, Abdul-Khaliq H, Alastalo TP, Beerbaum P, Bonnet D, et al. Executive summary. Expert consensus statement on the diagnosis and treatment of paediatric pulmonary hypertension. The European paediatric pulmonary vascular disease network, endorsed by ISHLT and DGPK. Heart. 2016; 102(Suppl 2):ii86-100.

6. Beghetti M. Fontan and the pulmonary circulation: a potential role for new pulmonary hypertension therapies. Heart. 2010;96:911-6.

7. Ciliberti P, Schulze-Neick I, Giardini A. Modulation of pulmonary vascular resistance as a target for therapeutic interventions in Fontan patients: focus on phosphodiesterase inhibitors. Future Cardiol. 2012;8:271-84.

8. Tunks RD, Barker PC, Benjamin DK Jr, Cohen-Wolkowiez M, Fleming GA, Laughon M, et al. Sildenafil exposure and hemodynamic effect after Fontan surgery. Pediatr Crit Care Med. 2014;15:28-34.

9. Downing TE, Whitehead KK, Dori Y, Gillespie MJ, Harris MA, Fogel MA, et al. Accuracy of conventional oximetry for flow estimation in patients with superior cavopulmonary connection: a comparison with phase-contrast cardiac MRI. Circ Cardiovasc Imaging. 2013;6:943-9.

10. Latus H, Gerstner B, Kerst G, Moysich A, Gummel K, Apitz C, et al. Effect of inhaled nitric oxide on blood flow dynamics in patients after the Fontan procedure using cardiovascular magnetic resonance flow measurements. Pediatr Cardiol. 2016;37:504-11.

11. Celermajer DS, Cullen S, Deanfield JE. In vivo detection of endothelium dependent and independent pulmonary artery relaxation in children. Br Heart J. 1993; 69:298-302.

12. Celermajer DS, Cullen S, Deanfield JE. Impairment of endothelium-dependent pulmonary artery relaxation in children with congenital heart disease and abnormal pulmonary hemodynamics. Circulation. 1993;87:440-6.

13. Zimmermann R, Kreuder J, Michel-Behnke I, Voelkel NF, Schranz D. Pulmonary flow reserve in children with idiopathic pulmonary arterial hypertension: implications for diagnosis and therapy. Eur J Med Res. 2006;11:208-13.

14. Apitz C, Zimmermann R, Kreuder J, Jux C, Latus H, Pons-Kuhnemann J, et al. Assessment of pulmonary endothelial function during invasive testing in children and adolescents with idiopathic pulmonary arterial hypertension. J Am Coll Cardiol. 2012;60:157-64.

15. Latus H, Werz A, Kock I, Rupp S, Kerst G, Kreuder J, et al. Systemic arterial endothelial function in children and young adults with idiopathic pulmonary arterial hypertension: is there a relation to pulmonary endothelium-dependent relaxation? Pediatr Cardiol. 2014;35:844-50.

16. Latus H, Wagner I, Ostermayer S, Kerst G, Kreuder J, Schranz D. Hemodynamic evaluation of children with persistent or recurrent pulmonary arterial hyperten- sion following complete repair of congenital heart disease. Pediatr Cardiol. 2017:38:1342-9.

17. Ilsar R, Chawantanpipat C, Chan KH, Dobbins TA, Waugh R, Hennessy A, et al Doppler-derived pulmonary flow reserve detects pulmonary microvascular obstruction in high primates. Heart Lung Circ. 2010;19:592-4.

18. Celermajer DS, Dollery C, Burch M, Deanfield JE. Role of endothelium in the maintenance of low pulmonary vascular tone in normal children. Circulation. 1994;89:2041-4.

19. Ong P, Athanasiadis A, Sechtem U. Patterns of coronary vasomotor responses to intracoronary acetylcholine provocation. Heart. 2013;99:1288-95.

20. Rocha AM, Salemi VM, Lemos Neto PA, Matsumoto AY, Pereira VF, Fernandes F, et al. Endothelial and non-endothelial coronary blood flow reserve and left ventricular dysfunction in systemic hypertension. Clinics (Sao Paulo). 2009;64:327-35.

21. Henaine R, Vergnat M, Bacha EA, Baudet B, Lambert V, Belli E, et al. Effects of lack of pulsatility on pulmonary endothelial function in the Fontan circulation. $J$ Thorac Cardiovasc Surg. 2013;146:522-9.

22. Ridderbos FJ, Wolff D, Timmer A, et al. Adverse pulmonary vascular remodeling in the Fontan circulation. J Heart Lung Transplant. 2015;34:404-13.

23. Latus H, Gummel K, Diederichs T, Bauer A, Rupp S, Kerst G, et al. Aortopulmonary collateral flow is related to pulmonary artery size and affects ventricular dimensions in patients after the Fontan procedure. PLoS One 2013:8:e81684.

24. Conraads VM, Bosmans JM, Claeys MJ, Vrints CJ, Snoeck JP, De Clerk L, et al. Paradoxic pulmonary vasoconstriction in response to acetylcholine in patients with primary pulmonary hypertension. Chest. 1994;106: 385-90.

25. Porter TR, Taylor DO, Cycan A, Fields J, Bagley CW, Pandian NG, et al. Endothelium-dependent pulmonary artery responses in chronic heart failure: influence of pulmonary hypertension. J Am Coll Cardiol. 1993;22:1418-24.

26. Cherry PD, Gillis CN. Evidence for the role of endothelium-derived relaxing factor in acetylcholine-induced vasodilatation in the intact lung. J Pharmacol Exper Ther. 1987;241:516-20.

27. Hyman AL, Kadowitz PJ. Tone-dependent responses to acetylcholine in the feline pulmonary vascular bed. J Appl Physiol (1985). 1988;64: 2002-9.

28. Ilsar R, Chawantanpipat C, Chan KH, Dobbins TA, Waugh R, Hennessy A, et al. Measurement of pulmonary flow reserve and pulmonary index of microcirculatory resistance for detection of pulmonary microvascular obstruction. PLoS One. 2010;5:e9601.

Key Words: Fontan circulation, pulmonary vascular remodeling, pulmonary vasoreactivity 
TABLE E1. Demographic, clinical, and hemodynamic characteristics of 3 patients who showed a paradoxical increase in local pulmonary vascular resistance (PVR) during acetylcholine (Ach) infusion

\begin{tabular}{|c|c|c|c|}
\hline Variable & Patient 1 & Patient 2 & Patient 3 \\
\hline Sex & Male & Female & Male \\
\hline Diagnosis & HLHS & HLHS & HLHS \\
\hline Age at study (y) & 15.9 & 4.1 & 9.6 \\
\hline Age at total cavopulmonary connection (y) & 3.2 & 2.6 & 3.8 \\
\hline New York Heart Association functional class & III & III & III \\
\hline Transcutaneous oxygen saturation (\%) & 90 & 93 & 93 \\
\hline Fenestration & No & No & No \\
\hline Mean pulmonary artery pressure (mm $\mathrm{Hg}$ ) & 12 & 13 & 9 \\
\hline Pulmonary capillary wedge pressure $(\mathrm{mm} \mathrm{Hg})$ & 5 & 9 & 4 \\
\hline Transpulmonary gradient (mm Hg) & 7 & 4 & 5 \\
\hline Global indexed pulmonary vascular resistance $\left(\mathrm{WU} \times \mathrm{m}^{2}\right)$ & 4.4 & 0.7 & 2.3 \\
\hline Average peak velocity baseline $(\mathrm{cm} / \mathrm{s})$ & 17 & 30 & 11 \\
\hline Average peak velocity Ach $(\mathrm{cm} / \mathrm{s})$ & 19 & 25 & 11 \\
\hline Pulmonary flow reserve & 1.1 & 0.8 & 1.0 \\
\hline Distal pulmonary artery pressure baseline $(\mathrm{mm} \mathrm{Hg})$ & 9 & 14 & 8 \\
\hline Distal pulmonary artery pressure $\mathrm{Ach}(\mathrm{mm} \mathrm{Hg})$ & 11 & 15 & 9 \\
\hline Local PVR baseline $(\mathrm{mm} \mathrm{Hg} / \mathrm{cm} / \mathrm{s})$ & 0.52 & 0.47 & 0.71 \\
\hline Local PVR Ach $(\mathrm{mm} \mathrm{Hg} / \mathrm{cm} / \mathrm{s})$ & 0.59 & 0.61 & 0.80 \\
\hline
\end{tabular}

HLHS, Hypoplastic left heart syndrome; $P V R$, pulmonary vascular resistance. 